\title{
The herbal compound Songyou Yin (SYY) inhibits hepatocellular carcinoma growth and improves survival in models of chronic fibrosis via paracrine inhibition of activated hepatic stellate cells
}

\author{
Yang $\mathrm{Bu}^{1,2, *}$, Qing-An $\mathrm{Jia}^{3, *}$, Zheng-Gang Ren $^{2, *}$, Tong-Chun Xue ${ }^{2}$, Quan-Bao \\ Zhang2, Ke-Zhi Zhang', Qiang-Bo Zhang', Yang You², Hui Tian², Lun-Xiu Qin ${ }^{3}$, \\ Zhao-You Tang ${ }^{2}$ \\ ${ }^{1}$ Hepatobiliary Surgery, General Hospital of Ningxia Medical University, Yinchuan 750001, China \\ ${ }^{2}$ Liver Cancer Institute, Zhongshan Hospital, Fudan University, Key Laboratory of Carcinogenesis and Cancer Invasion, \\ Ministry of Education, Shanghai 200032, China \\ ${ }^{3}$ Cancer Center, Institutes of Biomedical Sciences, General Surgery, Huashan Hospital, Fudan University, Shanghai 200032, \\ China \\ *These authors have contributed equally to this work \\ Correspondence to: \\ Zhao-You Tang, e-mail: zytang88@163.com \\ Keywords: Songyou Yin, hepatocellular carcinoma, chronic fibrosis, activated hepatic stellate cells \\ Received: April 08, $2014 \quad$ Accepted: October 09, $2015 \quad$ Published: October 22, 2015
}

\section{ABSTRACT}

Chronic fibrosis is a major risk factor for the development of hepatocellular carcinoma (HCC). The pathological progression of hepatic fibrosis has been linked to cellular processes that promote tumor growth and metastasis. Several recent studies have highlighted the cross-talk between tumor cells and activated hepatic stellate cells (aHSCs) in HCC. The herbal compound Songyou Yin (SYY) is known to attenuate hepatoma cell invasion and metastasis via down-regulation of cytokine secretion by aHSCs. However the underlying mechanism of SYY treatment in reversal of hepatic fibrosis and metastasis of liver cancers is not known. In the current study, a nude mouse model with liver fibrosis bearing orthotopic xenograft was established and we found that SYY could reduce associated fibrosis, inhibit tumor growth and improve survival. In the subcutaneous tumor model with fibrosis, we found that SYY could inhibit liver cancer. In vitro, hepatoma cells incubated with conditioned media (CM) from SYY treated aHSCs showed reduced proliferation, decrease in colony formation and invasive potential. SYY treated group showed altered gene expression, with 1205 genes up-regulated and 1323 genes down-regulated. Gene cluster analysis indicated that phosphatidylinositol-3-kinase (PI3K) was one of the key genes altered in the expression profiles. PI3K related markers were all significantly down-regulated. ELISA also indicated decreased secretion of cytokines which were regulated by PI3K/AKT signaling after SYY treatment in the hepatic stellate cell line, LX2. These data clearly demonstrate that SYY therapy inhibits HCC invasive and metastatic potential and improves survival in nude mice models with chronic fibrosis background via inhibition of cytokine secretion by activated hepatic stellate cells.

\section{INTRODUCTION}

Liver cancer (mainly hepatocellular carcinoma $[\mathrm{HCC}]$ ), ranks as the fifth most common cancer and worldwide it is the second most frequent cause of death in men [1]. In China, it is observed that chronic hepatitis followed by concomitant liver cirrhosis is the predominant risk factor for the development of HCC [2]. A previous study has demonstrated that activated hepatic stellate cells (aHSCs) play a key role in causing liver cirrhosis and infiltration into the HCC stroma, which supports malignancy progression [3]. 
Some reports have shown that in response to liver damage from viruses, alcohol, drug abuse and any other cause, the aHSCs trans-differentiate into myofibroblasts (MFs), which leads to hepatic fibrosis or even cirrhosis $[4,5]$. Several recent studies have indicated that cross-talk occurs between tumor cells and aHSCs during progression of HCC [6, 7]. Wang and colleagues [8] reported that cancer associated fibroblasts (CAFs) isolated from lung cancer tissue produces hepatocyte growth factor (HGF), which activates the c-Met pathway resulting in the invasion and metastasis of cancer cells [9]. Another study reported that fibroblasts isolated from breast cancer tissue enhances cancer cell invasiveness through an interleukin-6 (IL-6)-dependent signaling mechanism [10]. Thus, there is evidence to support the concept that aHSCs contribute to the development and progression of different types of malignancies. Similarly, aHSCs enhance hepatoma cell invasiveness and metastasis through paracrine signaling mechanisms involving cytokine secretion $[11,12]$. Furthermore, the transforming growth factor- $\beta$ (TGF- $\beta$ ) receptor inhibitor, LY2109761, was shown to inhibit the production of TGF- $\beta$ secretion by CAFs, which blocked the cross-talk between CAFs and HCC and inhibited progression of HCC [13]. Supported by these previous studies, aHSCs are recognized critical players in the development and progression of malignancy $[14,15]$. Inhibition of aHSC activity in the tumor microenvironment represents a potential therapeutic strategy to prevent and treat HCC.

Since ancient times, herbal medicines have been used in China to treat different malignancies. [16]. Several studies have shown that extracts from traditional herbal medicines possess anticancer potential, warranting further study. These studies have shown that herbal extracts possess anticancer activity and can modulate cell cycle, apoptotic signaling, expression of angiogenic factors, invasion and metastatic potential in cancer cells [17-27]. The herbal medicine Bu-Zhong-Yi-Qi Tang is a mixture of ten herbs, and was shown to suppress the growth of hepatoma cells in vitro through the activation of a p53independent pro-apoptotic signaling mechanism [28]. Similarly, Sho-Saiko-To, which is a mixture of seven herbs, inhibited the proliferation of human hepatoma cells by inducing cell cycle arrest and apoptosis [29]. Our recent study with Songyou Yin (SYY), a mixture of five herbs, showed inhibition of HCC growth and prolonged survival in a mice model by inducing caspase-3-dependent tumor cell apoptosis [30]. We also demonstrated that SYY inhibited HCC invasiveness by down-regulation of enzyme matrix metalloproteinase-2 (MMP-2) [30]. Xiong et al., found that SYY could attenuate oxaliplatinenhanced HCC metastatic potential [31]. Jia et al., reported that SYY attenuated hepatoma cell invasiveness and metastasis in vitro via down-regulation of cytokines secreted by aHSCs [11]. However, whether SYY has a role in reversal of hepatic fibrosis and which signal transduction pathway leads to inhibition of hepatoma progression remains largely unknown.
In the present study, a mouse model with a fibrotic background was established to determine if SYY could attenuate hepatic fibrosis and block the cross-talk between aHSCs and HCC in xenograft tumors. We also investigated the ability of SYY to indirectly influence the malignancy potential and progression of hepatoma cells and the molecular mechanisms involved.

\section{RESULTS}

\section{Establishment of a nude mouse model with fibrosis}

In order to study the correlation between liver fibrosis and HCC, it is important to establish a stable mouse model with fibrosis. We utilized the method of subcutaneous injection of $\mathrm{CCl}_{4}$ and found, as expected, that the severity of hepatic fibrosis increased with the prolonged treatment of $\mathrm{CCl}_{4}$. The liver stiffness, the particles on the liver surface and volume are three of the important exterior characteristics in evaluating liver cirrhosis. We found the severity of hepatic fibrosis was increased by exhibiting the increased particles on the liver surface, and there was reduced liver volume with the prolonged treatment of $\mathrm{CCl}_{4}$ (Figure 1A). $\mathrm{H} \& \mathrm{E}$ and Sirius staining showed there was continuous collagen accumulation induced by prolonged $\mathrm{CCl}_{4}$ treatment (Figure 1B, 1C). $\alpha$-SMA, which is a marker of hepatic fibrosis, was also up-regulated after $\mathrm{CCl}_{4}$ treatment (Figure 1D).

\section{SYY inhibited tumor growth and reduced associated fibrosis in nude mice bearing orthotopic xenografts with a fibrosis background}

Based on the nude mouse model with fibrosis mentioned above, we further established the nude mouse model bearing orthotopic xenograft with fibrosis. These were divided in untreated and SYY treated groups. There was enhanced proliferation in the untreated group (HCCLM3 $+\mathrm{CCl}_{4} 2.418 \pm 0.24$ vs. HCCLM3 $1.748 \pm 0.15, P=0.0448)$. SYY $(2 \mathrm{~g} / \mathrm{kg} /$ day $)$ exhibited no significant inhibition of tumor growth (HCCLM3, 1.74 $8 \pm 0.15$ vs. HCCLM3 + SYY $1.736 \pm 0.13, P=0.9514)$ while, in the treated group induced by $\mathrm{CCl}_{4}$, the same dosage of SYY showed a significant inhibition of tumor growth $\left(\mathrm{HCCLM} 3+\mathrm{CCl}_{4} 2.418 \pm 0.2376\right.$ vs. HCCLM3 + $\mathrm{CCl}_{4}+\mathrm{SYY} 1.584 \pm 0.1725, P=0.0218$ ) (Figure 2A). $\mathrm{H} \& \mathrm{E}$ and Sirius staining highlighted the increased fibrous connective tissue in tumor stroma induced by $\mathrm{CCl}_{4}$ (Figure 2B, 2C). The expression of $\alpha-\mathrm{SMA}$, also increased (HCCLM3 $1259 \pm 112.2$ vs. $\mathrm{HCCLM} 3+\mathrm{CCl}_{4}$ $12180 \pm 1073, P=0.0006)$ in untreated group, but was down-regulated in SYY treated group (HCCLM3 $+\mathrm{CCl}_{4}$ $12180 \pm 1073$ vs. HCCLM3 $+\mathrm{CCl}_{4}+\mathrm{SYY} 7327 \pm 476.3$, $P=0.0144)$ (Figure 2D). 


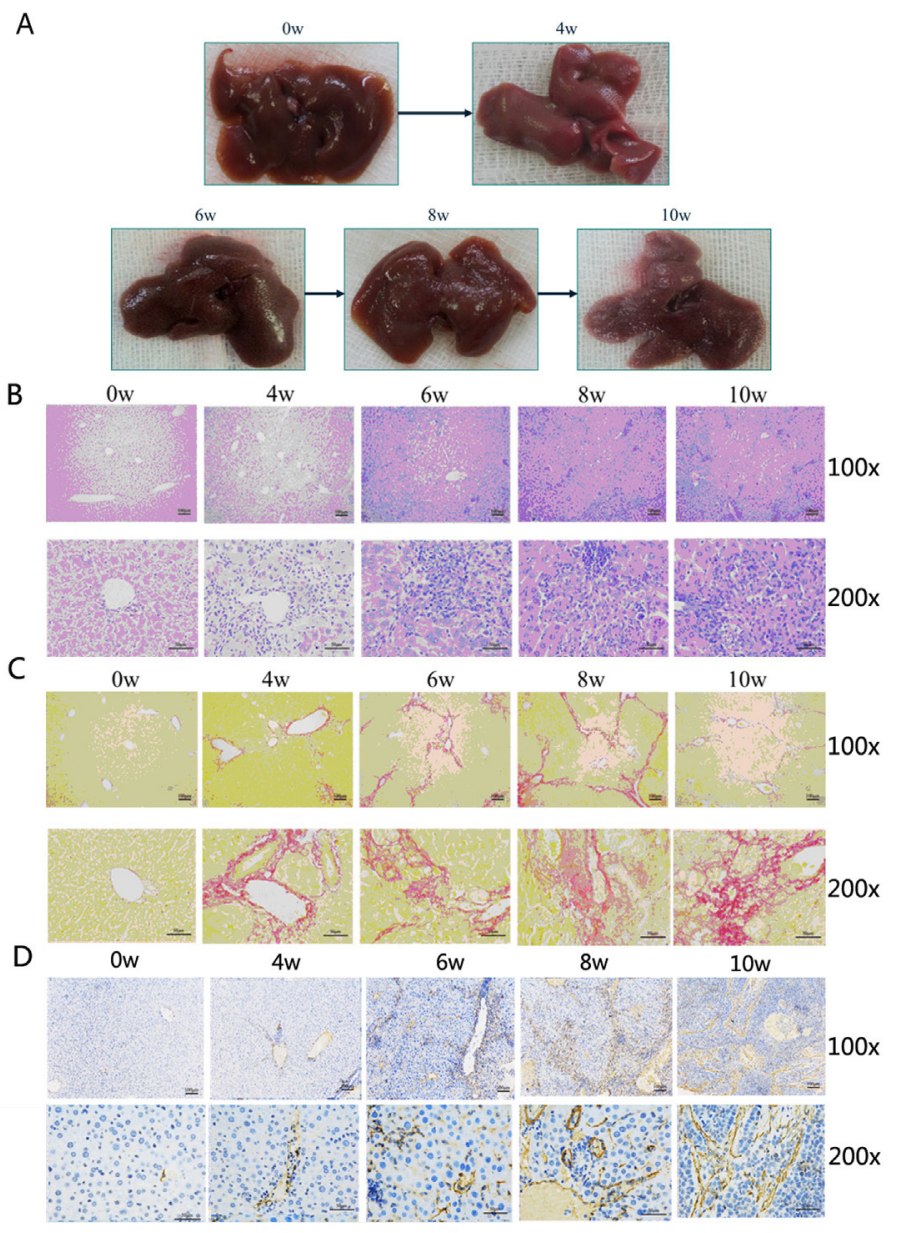

Figure 1: The nude mouse model with cirrhosis induced by carbon tetrachloride $\left(\mathrm{CCL}_{4}\right)$ was successfully established. A. The increase in severity of hepatic fibrosis was observed as the presence of more number of particles on the liver surface with reduction in liver volume with the prolonged treatment with $\mathrm{CCL}_{4}$. B. $\mathrm{H} \& \mathrm{E}$ staining exhibited the same tendency of increased $\mathrm{f}$ hepatic fibrosis with increased fibrous connective tissue. C. Sirius staining also showed continuous collagen accumulation induced by prolonged $\mathrm{CCL}_{4}$ treatment. D. $\alpha$-SMA, which is a marker of hepatic fibrosis, was also up-regulated after treatment with $\mathrm{CCl}_{4}$.

\section{SYY inhibited HCC growth, reduced associated fibrosis and prolonged survival in the xenograft tumor model with fibrosis background}

The nude mouse xenograft model with a fibrosis background was established and the correlation between tumor parenchymal cells and aHSCs was evaluated. The HCCLM3 cell density of $5 \times 10^{4}$ and $1 \times 10^{5}$ could not form xenograft tumors. Even with the total number of cells approaching $5 \times 10^{5}$, only half of the xenograft tumors were formed. While HCCLM3 with cell density of $1 \times 10^{6}$ could form full xenograft tumors (Figure 3A, Table 1). The mixture of $5 \times 10^{4}$ HCCLM 3 and $1 \times 10^{5}$ aHSCs, formed xenograft tumors. The volume of the xenograft tumors were related to the number of HCCLM3 cells used (Figure 3B, Table 1). For further studies, mixture of $5 \times 10^{5}$ HCCLM 3 cells and $5 \times 10^{5}$ aHSCs cells was used to form xenograft tumors. There was significant reduction in the tumor volume after treatment with SYY $($ HCCLM3 + HSC $2.36 \pm 0.21 v s$. HCCLM3 + HSC + SYY $1.12 \pm 0.17, P=0.0331$ ) (Figure 3C). PCNA, which is a marker of proliferation, was down-regulated in tumor tissues in SYY treated group (HCCLM3 + HSC $22880 \pm 1510 v s$. HCCLM3 + HSC + SYY 15240 $\pm 1050, P=0.0143)$. The expression of $\alpha$-SMA was also down-regulated after treatment with SYY in the LM3 and HSC mixed tissues (HCCLM3 + HSC $25830 \pm 1269$ $v s$. HCCLM3 + HSC + SYY $14910 \pm 1057, P=0.0027)$ (Figure 3D). H\&E and Sirius staining indicated reduced fibrous tissue and collagen accumulation in tumor tissues after SYY treatment (Figure 3E). Finally, in aHSCs, $\alpha$-SMA was significantly decreased as demonstrated by immunofluorescence (red staining) (Figure 3F). Meanwhile, the SYY treatment group in the xenograft model showed that hepatic cancer cells were attenuated in metastasizing to the lungs (HCCLM3 + HSC $7.0 \pm 1.0 v s$. 


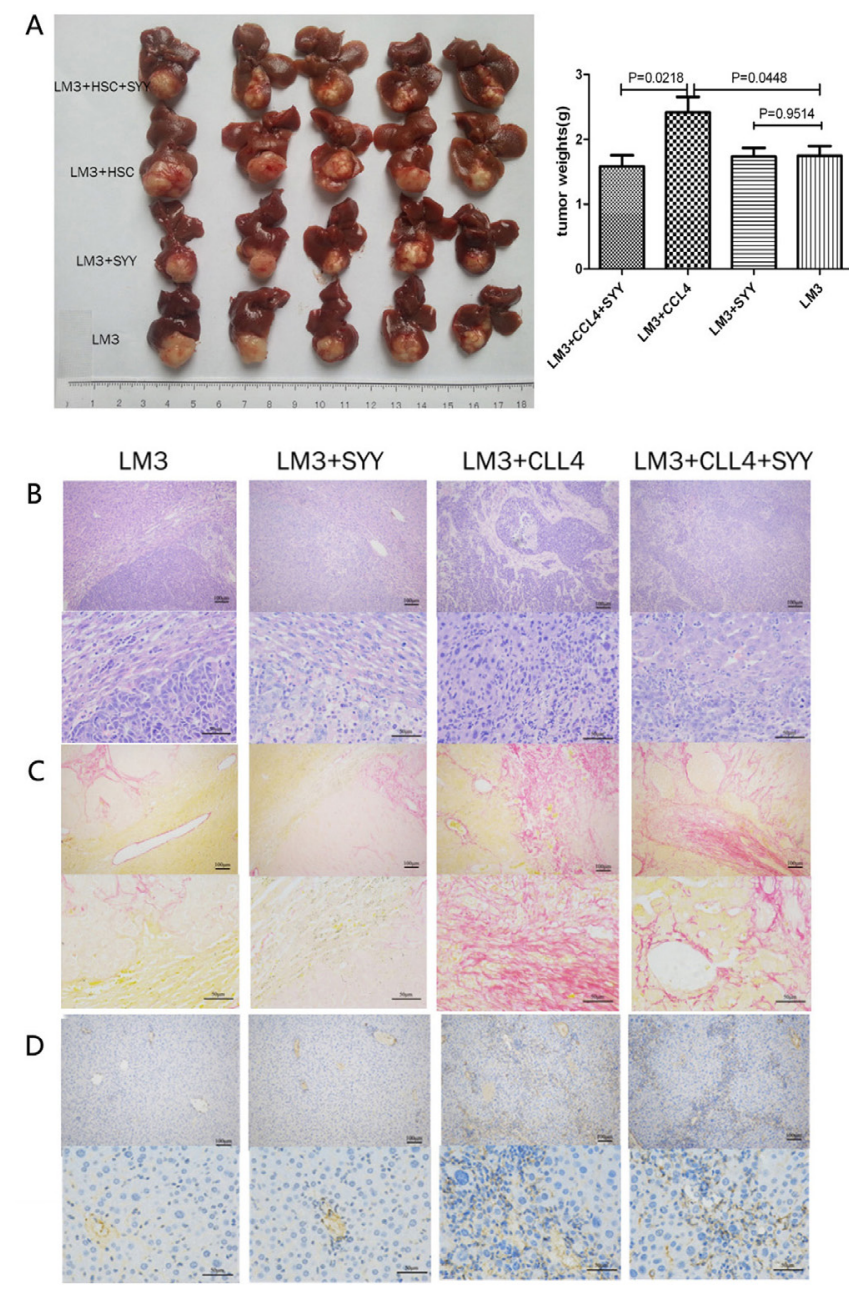

Figure 2: The nude mouse model bearing orthotopic xenografts was established. The orthotopic tumors in the models with cirrhosis background were larger than tumors in the models with normal liver tissue background. Treatment of tumors with cirrhosis background with SYY significantly reduced tumor volume. A. There were no significant changes in tumor volume with normal liver tissue background. B. H\&E staining exhibited reduced fibrous connective tissue in the tumor stroma. C. Sirius staining also showed that reduced collagen accumulation and D. down-regulated expression of $\alpha$-SMA in tumor stroma were all related to SYY treatment.

HCCLM3 + HSC + SYY $1.4 \pm 0.51, P=0.0011)$ and led to prolonged survival (HCCLM3 + HSC $51.33 \pm 5.26$ days vs. HCCLM3 + HSC + SYY $63.17 \pm 9.22$ days, $P=0.0016$ ) (Figure 4).

\section{Hepatoma cells treated with CM from SYY pretreated aHSCs showed reduced malignant potential}

While studying the basic characteristics of SYY, we found that SYY exhibited concentration-dependent inhibition. Hence we chose SYY $(8 \mathrm{mg} / \mathrm{ml})$ as the final concentration for further experiments, which exhibited no significant cytotoxicity in LX2 (Supplementary materials 1). The clonogenic capacity of HCCLM3 and Hep3B was enhanced after treatment with aHSCs CM $($ HCCLM3 $1.853 \pm 0.0867 v s$. HCCLM3 + CM-nSYY $4.000 \pm 0.1155, P<0.0001 ;$ Hep3B $2.100 \pm 0.0547$ vs. Hep3B + CM-nSYY $4.800 \pm 0.1201, P<0.0001)$.
SYY showed no direct inhibition on the clonogenic capacity of HCCLM3 and Hep3B (HCCLM3 1.853 \pm 0.0867 vs. HCCLM3 + SYY $1.947 \pm 0.0866, P=0.4888$; Hep3B $2.100 \pm 0.0547 v s$. Hep3B + SYY $2.300 \pm 0.0512$, $P=0.0705$ ). However, the CM from SYY treated aHSCs significantly inhibited the clonogenic capacity of LM3 and Hep3B (HCCLM3 + CM-SYY $4.400 \pm 0.1155$ vs. HCCLM3 + CM-nSYY $1.127 \pm 0.0636, P<0.0001$; Hep3B + CM-SYY $4.800 \pm 0.1201 v s$. Hep3B + CM-nSYY $1.400 \pm 0.0577, P<0.0001$ ) (Figure 5A). The number of HCCLM3 and Hep3B cells passing through the basement membrane was increased after treatment with $\mathrm{CM}$ obtained from aHSCs (HCCLM3 31.00 $\pm 1.528 v s$. HCCLM3 + CMnSYY $67.33 \pm 1.764, P<0.0001$; Hep3B $46.67 \pm 1.764$ $v s$. Hep3B + CM-nSYY $84.00 \pm 3.215, P=0.0005)$. SYY showed no direct inhibition on the number of HCCLM3 and Hep3B cells passing through the basement membrane (HCCLM3 $31.00 \pm 1.528$ vs. HCCLM3 + SYY $32.33 \pm 1.453, P=0.5614$; Hep3B $46.67 \pm 1.764 v s$. 

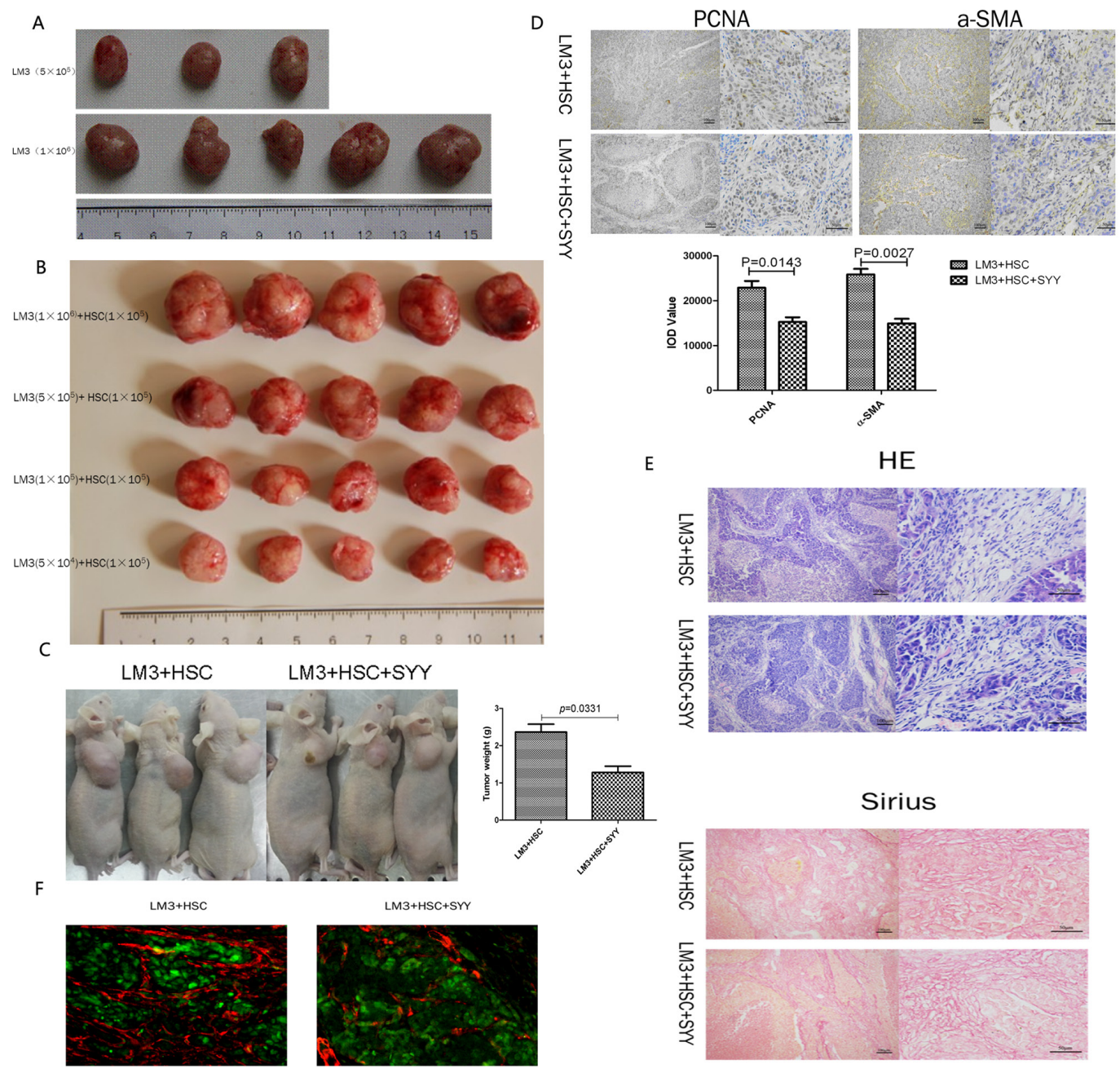

Figure 3: Subcutaneous tumor model of liver cancer was established and the correlation among tumor parenchymal cells, aHSCs in the stroma and SYY were investigated. LM3 in the range of $5 \times 10^{4}$ to $1 \times 10^{5}$ cells did not form tumors, and even with the total number of cells approaching $5 \times 10^{5}$ only half of the amount of tumors were formed A. LM3 in the number of $5 \times 10^{4}$ can form tumors when added with $1 \times 10^{5}$ aHSCs. The volumes of tumors were related to the number of LM3 cells B. Thus, $5 \times 10^{5}$ LM3 cells with $5 \times 10^{5}$ aHSCs were chosen to form tumors and the tumor volume was significantly reduced after treatment with SYY C. PCNA and $\alpha$-SMA were both down-regulated in tumor tissues after treatment with SYY D. HE and Sirius staining exhibited reduced fibrous connective tissue and collagen accumulation in tumor tissues after treatment with SYY E. Finally, aHSCs, which were marked by $\alpha$-SMA, were significantly decreased, as evident by immunofluroescence $\mathbf{F}$.

Hep3B + SYY $47.00 \pm 2.517, P=0.9189)$. We did find that the CM from SYY treated aHSCs significantly inhibited the number of HCCLM3 and Hep3B migrating cells (HCCLM3 + CM-nSYY $67.33 \pm 1.764$ vs. HCCLM3 + CM-SYY $23.33 \pm 0.8819, P<0.0001 ;$ Hep3B + CM-nSYY $84.00 \pm 3.215$ vs. Hep3B + CM-SYY $34.00 \pm 2.082$, $P=0.0002)$ (Figure 5B).

The cell proliferation of HCCLM3 and Hep3B was enhanced after treatment with CM from aHSCs (HCCLM3 $1.296 \pm 0.0211 v s$. HCCLM3 + CM-nSYY $2.391 \pm 0.0888$, $P<0.0001 ;$ Hep3B $1.131 \pm 0.0301$ vs. Hep3B + CM-nSYY $1.606 \pm 0.0837, P=0.0003)$. SYY showed no direct inhibition on the proliferative capacity of HCCLM3 and Hep3B (HCCLM3 $1.296 \pm 0.0211$ vs. HCCLM3 + SYY $1.310 \pm 0.0339, P=0.7280 ;$ Hep3B 1.131 $\pm 0.0301 v s$. Hep3B + SYY $1.214 \pm 0.0521, P=0.1967)$.
While CM from SYY treated aHSCs significantly inhibited the proliferative capacity of HCCLM3 and Hep3B (HCCLM3 + CM-nSYY $2.391 \pm 0.0888$ vs. HCCLM3 +CM-SYY $1.042 \pm 0.0569, P<0.0001$; Hep3B + CM-nSYY $1.606 \pm 0.0837 v s$. Hep3B + CM-SYY $0.5258 \pm 0.0149, P<0.0001$ ) (Figure 5C).

\section{SYY treatment altered gene expression and cytokine secretion of aHSCs}

There was difference between gene expression profiles of SYY treated LX2 cells and those of the treated controls. There were 1205 up-regulated genes and 1323 down-regulated genes following treatment of LX2 with SYY. Analysis of differential gene expression and signaling pathways indicated that PI3K was the key altered gene. 
Table 1: aHSCs promote the growth of HCC

\begin{tabular}{|l|l|l|}
\hline HCCLM3 & \multicolumn{2}{c}{ LX2 $\left(\mathbf{1} \times \mathbf{1 0}^{\mathbf{5}} / \mathbf{0 . 1} \mathrm{ml}\right)$} \\
\hline & $(-)$ & $(+)$ \\
\hline $1 \times 10^{6} / 0.1 \mathrm{ml}$ & $5 / 5$ & $5 / 5$ \\
\hline $5 \times 10^{5} / 0.1 \mathrm{ml}$ & $3 / 5$ & $5 / 5$ \\
\hline $1 \times 10^{5} / 0.1 \mathrm{ml}$ & $0 / 5$ & $5 / 5$ \\
\hline $5 \times 10^{4} / 0.1 \mathrm{ml}$ & $0 / 5$ & $5 / 5$ \\
\hline
\end{tabular}
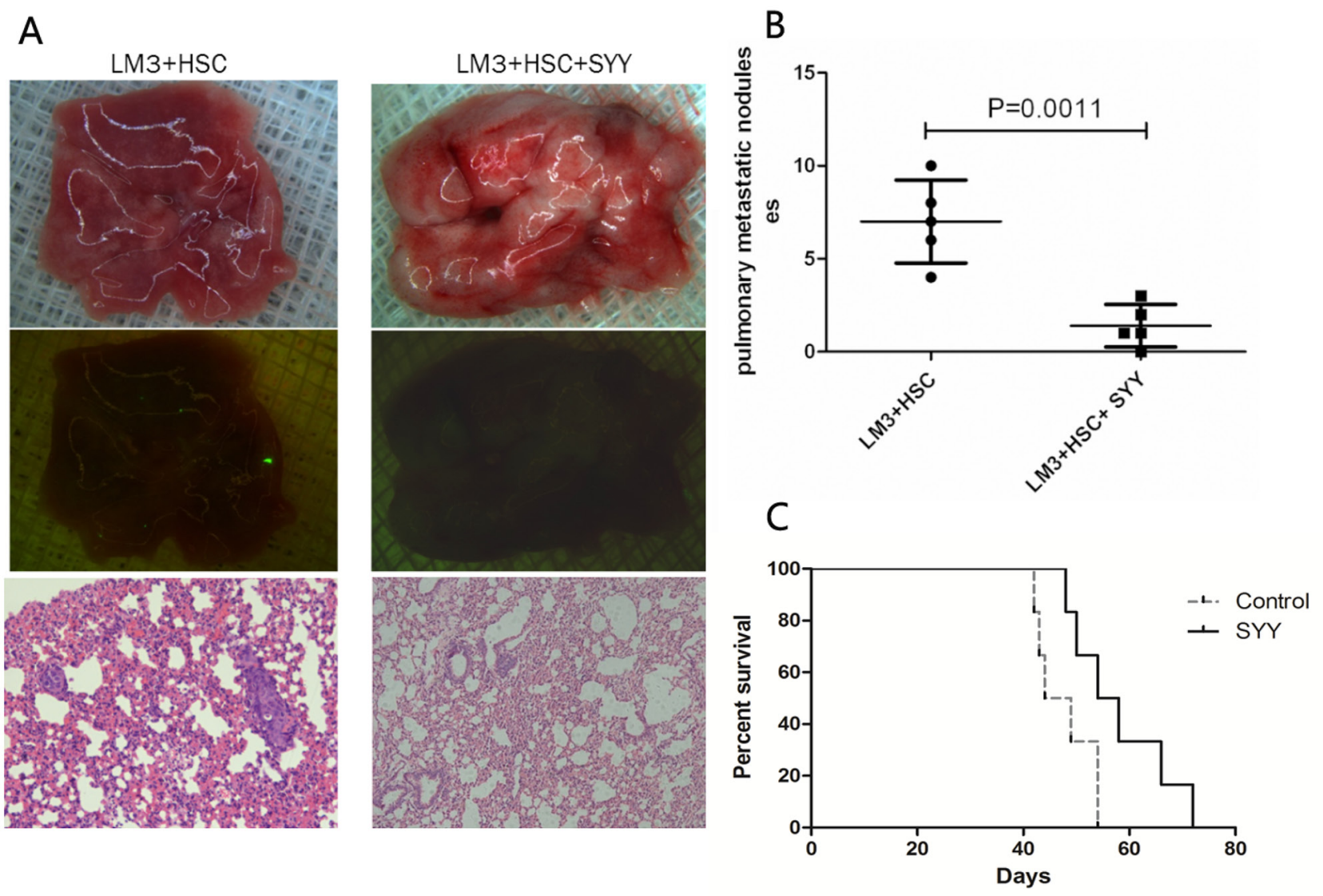

Figure 4: Reduced lung metastasis and prolonged survival were found in the orthotopic nude mouse models with cirrhosis background after treatment with SYY.

The Western blot analysis of proteins in SYY treated LX2 cells revealed that PI3K related signaling factors were all significantly down-regulated, including PI3K, AKT, pAKT, IGF1R, EGFR, TGF- $\beta 1$, VEGF, PDGF and MMP-2. (Figure 6A, 6B, 6C). Further, ELISA method showed that was significant decreased secretion of cytokines and other factors which were regulated by PI3K/AKT signaling after SYY treatment in LX2, such as TGF $\beta 1$ (CM-nSYY $32500 \pm 143.5$ vs. CM-SYY $26350 \pm 127.3$, $P<0.0001)$, MMP-2 (CM-nSYY $141500 \pm 15710$ vs. CM-SYY $92100 \pm 951.2, P=0.0350)$, HGF (CM-nSYY $9382 \pm 314.5$ vs. CM-SYY $3511 \pm 349.2, P=0.0003)$ and VEGF (CM-nSYY $1046 \pm 89.40$ vs. CM-SYY $664.6 \pm 29.14, P=0.0154)$. The additional cytokines altered significantly after SYY treatment of LX2 cells, were IL-10 (CM-nSYY $95.42 \pm 1.379$ vs. CM-SYY $134.7 \pm 1.42$, $P<0.0001)$, IL-12 (CM-nSYY $40.81 \pm 2.52$ vs. CM-SYY $134.7 \pm 1.42, P<0.0001)$, IL-2 (CM-nSYY $28.48 \pm 1.04$ vs. CM-SYY $81.97 \pm 0.66, P<0.0001)$, PDGF (CM-nSYY $6.02 \pm 0.43 v s$. CM-SYY $2.14 \pm 0.88, P=0.0161)$, EGF (CM-nSYY $2.97 \pm 0.15$ vs. CM-SYY $2.52 \pm 0.16$, $P<0.0001)$ and TNF- $\alpha(\mathrm{CM}-\mathrm{nSYY} 2.82 \pm 0.44$ vs. CM-SYY $4.40 \pm 0.56, P=0.0897$ ) (Table 2).

\section{DISCUSSION}

Approximately $90 \%$ of $\mathrm{HCC}$ cases arise in patients with cirrhosis, which clearly indicates that liver fibrosis 
A
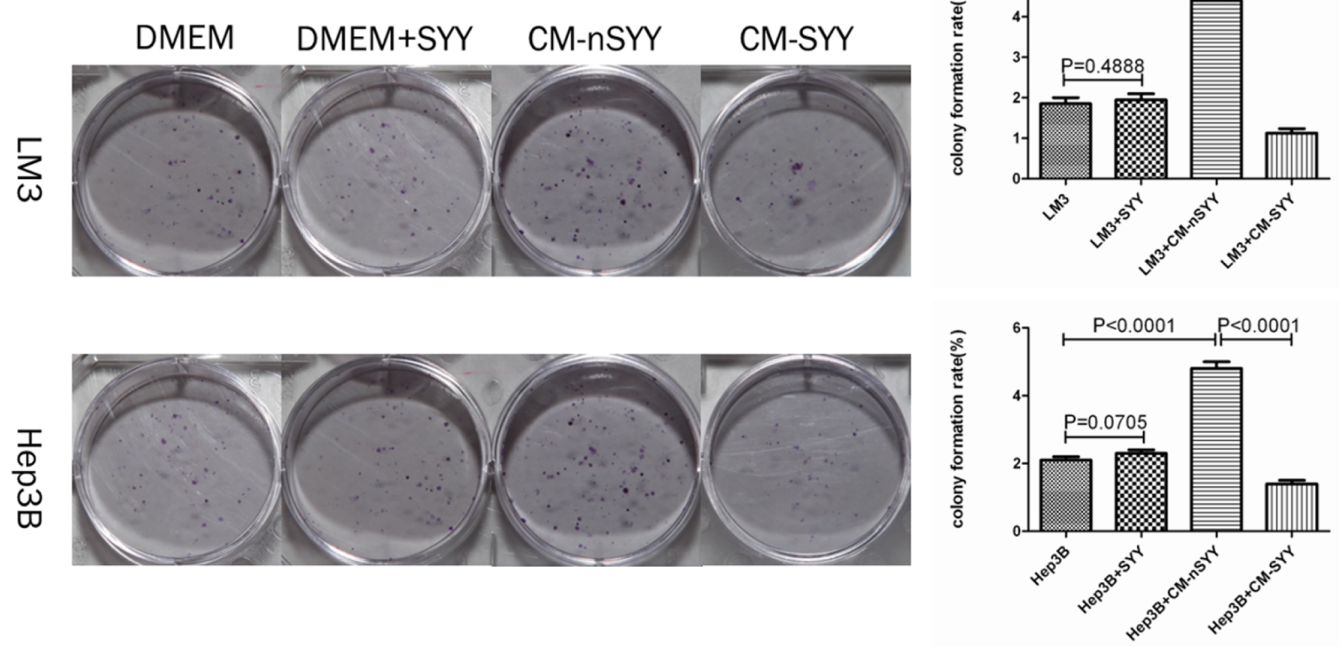

B
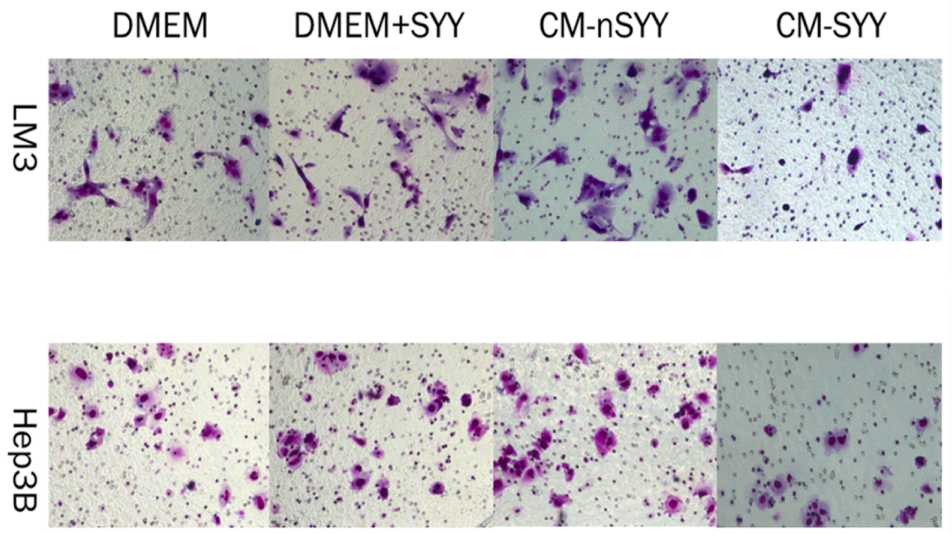

C

\section{LM3}

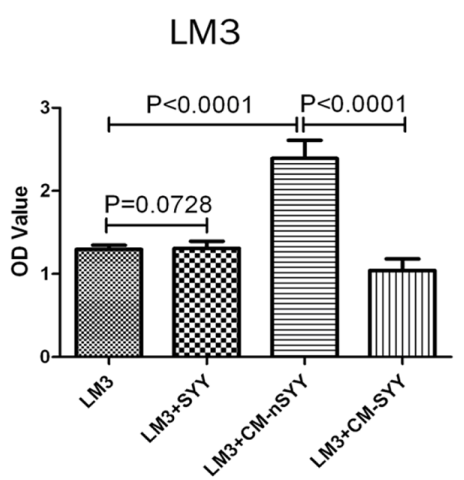

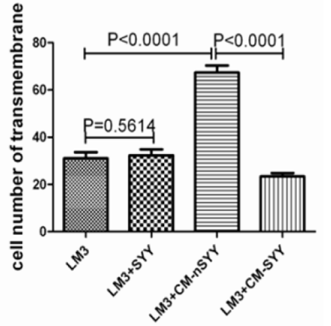
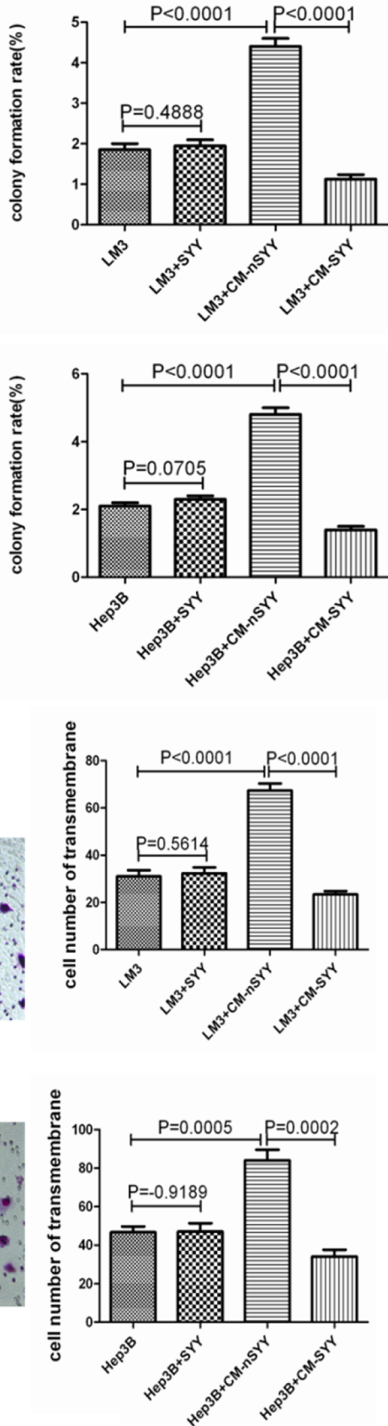

Hep3B

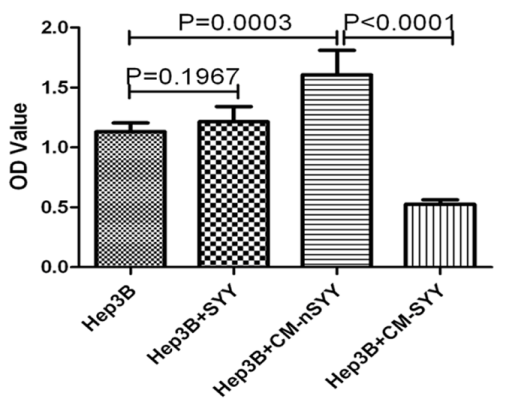

Figure 5: Clonogenic capacity of LM3 and Hep3B cells was enhanced after treatment with CM from aHSCs A. The transwell assays demonstrated that LM3 and Hep3B cells treated with CM from aHSCs passed through the basement membrane in larger number than cells in the control group. This effect was reduced by SYY treatment of cells exposed to aHSCs CM B. CCK8 assays showed that the proliferative capacity of LM3 and Hep3B was enhanced after treatment with CM from aHSCs, while SYY itself did not promote the proliferation of tumor cells $\mathbf{C}$.

is strongly associated with the development of HCC [33]. HSCs are known to be one of the key cell types that contribute to the pathogenesis of liver fibrosis. It is well known that HSCs are activated in response to liver damage and trans-differentiate into mesenchymal fibroblasts
(MFs), which play a key role in causing hepatic fibrosis. HSCs can also infiltrate the stroma of liver tumors as CAFs and further promote HCC cell proliferation and metastasis. It has been postulated that multiple signaling mechanisms may regulate aHSCs behavior which may 

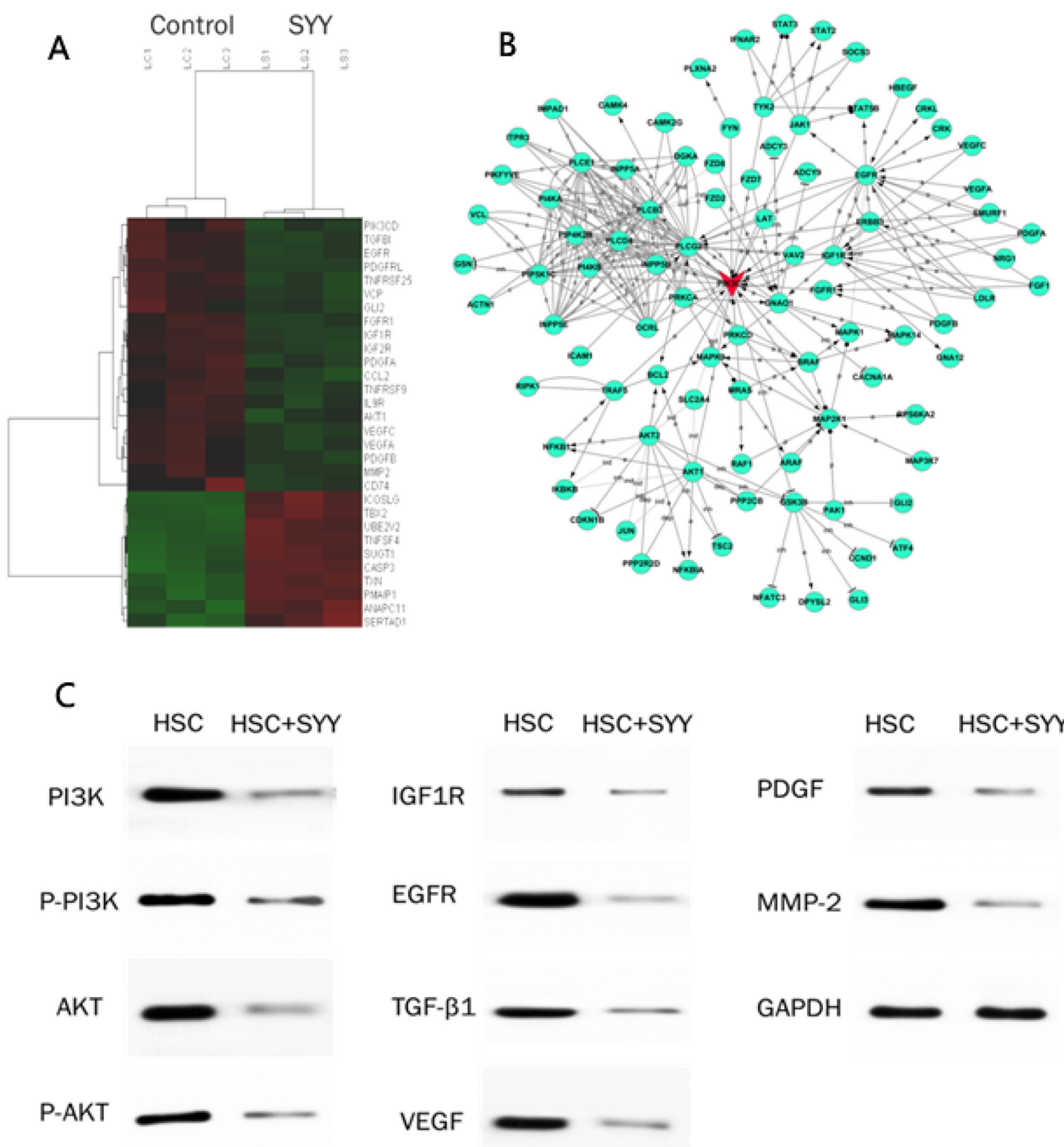

Figure 6: 1205 up-regulated and 1323 down-regulated gene were detected by Gene Microarray analysis, and the most relevant data are shown A. Analysis of differential gene expression and signaling pathways indicated that PI3K was the key gene in the altered gene profiles B. Western blots analysis showed that PI3K, pPI3k, AKT, pAKT, IGF1R, EGFR, TGF- $\beta$, VEGF, PDGF and MMP-2 were all down-regulated following SYY treatment $\mathbf{C}$.

induce changes in the hepatic tumor phenotype [34, 35]. A recent study has reported that aHSCs interact with hepatoma cells via secretion of cytokines, extracellular matrix (ECM)-mediated interactions and direct cell-to-cell contacts [36]. We previously reported that SYY indirectly attenuated hepatoma cell invasiveness and pulmonary metastasis through down-regulation of cytokines secreted by aHSCs [11]. However, the ability of SYY to reverse liver fibrosis and mechanisms regulating HCC inhibition remains largely unknown. Therefore, we chose a mouse model with a cirrhosis background to determine if SYY could attenuate hepatic fibrosis, block the cross-talk between aHSCs and HCC and indirectly influence the malignant potential of hepatoma cells.
Accumulating evidence supports the concept that aHSCs are the main matrix-producing cells in the process of liver fibrosis in response to liver damage $[4,5]$. Numerous studies have shown that aHSCs up-regulate the gene expression of ECM components, matrix-degrading enzymes and respective inhibitors, resulting in ECM remodeling and accumulation [37]. In the present study, we established a stable mouse model with a fibrosis background using chronic subcutaneous injection of $\mathrm{CCL}_{4}$. We found that the severity of hepatic fibrosis was increased by enhancing particles on the liver surface, and there was liver volume with the prolonged treatment of $\mathrm{CCL}_{4}$. As expected, H\&E and Sirius staining showed continuous collagen accumulation following $\mathrm{CCL}_{4}$ treatment. We also 
Table 2: Cytokine secretion of aHSCs after treatment with SYY

\begin{tabular}{|l|c|c|c|}
\hline \multicolumn{1}{l|}{ Cytokine } & CM-nSYY $(\mathbf{p g} / \mathbf{m l})$ & CM-SYY $(\mathbf{p g} / \mathbf{m l})$ & $\boldsymbol{P}$ value \\
\hline TGF- $\beta 1$ & $32500 \pm 143.5$ & $26350 \pm 127.3$ & $<0.0001$ \\
\hline MMP2 & $141500 \pm 15710$ & $92100 \pm 951.2$ & $<0.0350$ \\
\hline IL-10 & $95.42 \pm 1.379$ & $134.7 \pm 1.427$ & $<0.0001$ \\
\hline IL-12 & $40.81 \pm 2.524$ & $89.99 \pm 1.022$ & $<0.0001$ \\
\hline IL-2 & $28.48 \pm 1.0448$ & $81.97 \pm 0.6629$ & $=0.0001$ \\
\hline PDGF & $6.020 \pm 0.4255$ & $2.137 \pm 0.8838$ & $<0.0161$ \\
\hline EGF & $2.975 \pm 0.1520$ & $2.552 \pm 0.1587$ & $=0.0154$ \\
\hline VEGF & $1046 \pm 89.4$ & $664.6 \pm 29.14$ & $=0.0003$ \\
\hline HGF & $9382 \pm 314.5$ & $3511 \pm 349.2$ & $=0.0897$ \\
\hline TNF- $\alpha$ & $2.815 \pm 0.4392$ & $4.398 \pm 0.5574$ & \\
\hline
\end{tabular}

observed up-regulated $\alpha$-SMA expression following $\mathrm{CCL}_{4}$ treatment. An increasingly complex interplay between various cell types of the liver, stellate cells with tumor cells had now become apparent. [38, 39]. Therefore, based on the previous reports regarding nude mouse model with fibrosis as mentioned above, we further established a similar model bearing orthotopic xenograft in mice with fibrosis background.

The development of distant metastasis requires the invasion of cancer cells from the primary tumor into the surrounding tissue. To acquire such invasive abilities, cancer cells must undergo several phenotypic changes. aHSCs, is one of the most important members in the tumor microenvironment, which plays a critical role in modulating such phenotypic changes. Amann et al., [12] demonstrated that conditioned medium (CM) collected from aHSCs could promote proliferation and migration of HCC cells. There are many cytokines secreted by aHSCs that are associated with tumor invasion and metastasis, including HGF, EGF, IL-1, IL-2, IL-6, MMP-2, MMP-9, TGF- $\beta$, TNF- $\alpha$, VEGF and members of the Wnt signaling family $[9,39,40]$. Studebaker et al., [10] found soluble IL-6 produced by tissue-specific fibroblasts could promote growth and invasion of breast cancer cells, which could be inhibited by the removal or inhibition of IL-6. Li et al., [41] found that Wnt2, secreted by tumor fibroblasts, promoted tumor progression in oesophageal cancer by activation of the $\mathrm{Wnt} / \beta$-catenin signaling pathway. Recently, Jia et al., [11], found that SYY could attenuate hepatic cancer cell's invasive and metastatic capabilities by down-regulating cytokines secreted by aHSCs such as HGF, IL-6, TGF- $\beta$ and VEGF in vitro. In the present study, we found that SYY could have an effect on fibrosis and also could block the cross-talk between aHSCs and HCC.

SYY is a Chinese herbal medicine consisting of five herbs. Some components of SYY, such as Tanshinone and Astragalussaponins, have demonstrated efficacy in treatment of malignancies $[42,43]$. We have previously shown that SYY could effectively inhibit tumor growth and metastasis, reverse the molecular changes consistent with endothelial to mesenchymal transition and increase survival in a HCC nude mouse model $[30,31]$. In the present study, SYY inhibited liver cancer, reduced associated fibrosis and prolonged survival in the subcutaneous tumor model with fibrosis background. In addition, using an in vitro model, we also demonstrated that hepatoma cells treated by CM from SYY pretreated aHSCs had reduced malignant potential, with altered gene expression profile consisting of 1205 up-regulated and 1323 down-regulated genes. We found that PI3K was the key gene present in this altered gene profile. Further, protein analysis showed that the PI3K related markers such as PI3K AKT, pAKT, IGF1R, EGFR, TGF- $\beta 1$, VEGF, PDGF and MMP-2 were all down-regulated significantly after SYY treatment in LX2.

In the present study, we demonstrated that SYY could reduce liver cirrhosis and attenuate hepatic cancer cell invasiveness and metastasis through the downregulation of cytokines secreted by activated hepatic stellate cells. Our findings suggest that in future traditional Chinese medicines, such as SYY, may provide novel therapeutic approaches targeted to reduce liver cirrhosis and prevent liver cancers.

\section{MATERIALS AND METHODS}

\section{Cell lines and animals}

A highly metastatic human hepatocellular carcinoma cell line (HCCLM3), which originated from MHCC97H, was established as previously described [26, 27]. Hep3B from American Type Culture Collection was purchased from the Chinese Academy of Science. Male BALB/c nu/nu mice (aged 4-6 weeks and weighing approximately $20 \mathrm{~g}$ ) were obtained from the Chinese Academy of Science and maintained under standard pathogen-free conditions. The animal use protocol was approved by the Shanghai Medical Experimental Animal Care Commission. 


\section{Regents and antibodies}

Antibodies used for immunofluorescence, immunoblotting and/or immunohistochemistry were as follows: rabbit antihuman monoclonal $\alpha$-smooth muscle actin ( $\alpha$-SMA) (Abcam, Cambridge, MA, USA), rabbit anti-human monoclonal PI3K (Epitomics, Burlingame, CA, USA), rabbit antihuman monoclonal AKT (CST), rabbit antihuman monoclonal phosphorylated (p)-AKT (CST, Danvers, MA, USA), rabbit antihuman monoclonal insulin like growth factor-1 receptor (IGF1R) (Abcam), rabbit antihuman monoclonal epidermal growth factor receptor (EGFR) (Abcam), rabbit antihuman monoclonal transforming growth factor- $\beta 1$ (TGF- $\beta 1$ ) (Abcam), rabbit antihuman monoclonal vascular endothelial growth factor (VEGF) (Epitomics), rabbit antihuman monoclonal platelet derived growth factor (PDGF) (Epitomics), rabbit antihuman monoclonal MMP-2 (Epitomics) and rabbit antihuman monoclonal proliferating cell nuclear antigen (PCNA) (Epitomics).

\section{Characterization and preparation of herbal extracts}

The Chinese herbal medicine formula for SYY is a dietary compound approved by the Chinese State Food and Drug Administration (Grant No. G20070160) that includes five Chinese medicinal herbal extracts with a finger print of the following proportions $(\mathrm{w} / \mathrm{w})$ : Salviamiltiorrhiza Bge, 14.3\%; Astragalus membranaceus Bge, $14.3 \%$; Lycium barbarum L, 23.8\%; Crataegus pinnatifida Bge, 23.8\% and Trionyx sinensis Wiegmann, 23.8\% (all from China) [11]. SYY, with the same batch number (\#20110401), used in vitro was produced by Shanghai Fang Xin Pharmaceutical Technology Co., Ltd. (Shanghai, China). The $800 \mathrm{mg} / \mathrm{ml} \mathrm{SYY} \mathrm{preparation} \mathrm{was}$ sterilized twice by filtration with $0.22 \mu \mathrm{m}$ filter. (Millipore, Billerica, MA, USA). It was further prepared for in vitro use as described previously [32].

\section{Preparation of conditioned media $(\mathrm{CM})$}

The aHSCs were cultured in high glucose Dulbecco's modified eagle medium (DMEM) containing $10 \%(\mathrm{v} / \mathrm{v}) \mathrm{FBS}$ in $\mathrm{T}_{25}$ flasks $\left(1 \times 10^{5}\right.$ cells $)$ as previously described [11]. The next day, cultures were removed and $12 \mathrm{ml}$ fresh serum-free DMEM medium with SYY sample (2 $\mathrm{mg} / \mathrm{ml}$ ) was added to SYY group, and fresh DMEM medium was added to control sample (no SYY). $24 \mathrm{~h}$ later, the cultures were centrifuged at $800 \mathrm{rpm}$ and the supernatants were collected. All cell culture reagents were purchased from Invitrogen.

\section{Lactate dehydrogenase (LDH) cytotoxicity assay}

A cytotoxicity detection kit PLUS (Roche) that quantitates cytotoxicity by measuring lactic dehydrogenase (LDH) activity released from damaged cells. The aHSCs were cultured and removed from the plates with trypsin digestion when the cell density reached $80 \%$ confluence. The cells were then counted and pipetted into 96 -well plates at 1000 cells/well. The same plate contained background controls (medium only), low controls (spontaneous LDH release), high controls (maximum LDH release) and experimental samples ( $2 \mathrm{mg} / \mathrm{ml} \mathrm{SYY}$ ) which were prepared according to the manufacturer's instructions. These plates were incubated in an incubator at $37^{\circ} \mathrm{C}$ in $5 \% \mathrm{CO} 2$ for 4,8 , $12,24,48$ and $72 \mathrm{~h}$. Results were expressed as the mean absorbance of wells in groups measured at $492 \mathrm{~nm}$. Cytotoxicity (\%) was calculated using the equation: (experimental value - low control) / (high control - low control) $\times 100 \%$.

\section{Cell proliferation and colony formation assay}

aHSCs were cultured in 96-well plates $\left(3 \times 10^{3}\right.$ cells/well $)$ and exposed to SYY at increasing concentrations for 24, 48, 72 and $96 \mathrm{~h}$. Cell proliferation assays were carried out with the Cell Counting Kit 8 (CCK8; Dojindo) and absorbance (OD) of each well noted at $450 \mathrm{~nm}$. For colony formation assays, LM3 and Hep3B cells were cultured in 6 -well plates $\left(3 \times 10^{3}\right.$ cells $/$ well $)$ and cultured with DMEM and CM from aHSCs with or without SYY $(2 \mathrm{mg} / \mathrm{mL})$. Culture medium was replaced every 3 days, and the colonies were fixed with ice-cold $4 \%$ paraformaldehyde 14 days after the initiation of treatment. Cells were stained with Giemsa (Sigma) and photographed.

\section{Cell invasion assays}

Cell invasion of HCCLM3 and Hep3B cell lines were assessed by transwell assays (Boyden chambers; Corning). Briefly, $80 \mu \mathrm{l}$ matrigel (BD Biosciences) was added to the upper chamber $6 \mathrm{~h}$ prior to seeding the cells on the membrane. Subsequently, $6 \times 10^{4}$ cells were seeded into the upper chamber of each well of 24-well plates containing $8.0-\mu \mathrm{m}$ pore size membranes. Serumfree DMEM, with or without SYY and aHSCs CM with or without SYY were added to the lower chamber of each well. After $48 \mathrm{~h}$, cells that had crossed over to the underside of the membrane were visualized by staining with Giemsa (Sigma), and photographed at a magnification of $200 \mathrm{x}$.

\section{Immunofluorescence and western blot analysis}

The expression of markers PCNA and $\alpha$-SMA were determined by immunofluorescence. Fibrotic hepatic tissues were sliced, fixed, permeabilized, blocked and incubated with primary monoclonal antibodies overnight at $4^{\circ} \mathrm{C}$. Slides were washed and incubated with anti-rabbit Cy3-conjugated secondary antibody (Jackson). Cells were counterstained with 4-6-diamidino-2-phenylindole (DAPI) to visualize cell nuclei and detected by fluorescence 
microscopy (Olympus). The concentration of protein extracted from aHSCs and aHSCs-SYY was determined using BCA Protein Assay Kit (Beyotime).Western blot analysis of PI3K, AKT, pAKT, IGF1R, EGFR, TGF- $\beta 1$, VEGF, PDGF and MMP-2 protein expression was performed according to the manufacturer's instructions.

\section{Expression of DNA microarray and analysis}

DNA microarrays were used to evaluate changes in gene expression profiles of aHSCs after SYY treatment. Total RNA extracted from SYY treated aHSCs and control cells were used for DNA microarray analysis. Microarray analysis of three independent samples was performed using $4 \times 44 \mathrm{~K}$ human Genome Array chips (Taiwan Phalanxbiotech Gene Expression Profiling Microarray), according to the manufacturer's instructions. Data analysis was performed using Feature Extraction and bioinformatics software GENESPRING ver12.0

\section{Establishment of animal model and treatment procedure}

\section{Establishment of the nude mouse with liver fibrosis}

Male BALB/c nu/nu mice (4-6 weeks old; $n=30)$ received subcutaneous injection of $5 \mu \mathrm{l} / \mathrm{g}$ carbon tetrachloride $\left(\mathrm{CCl}_{4} /\right.$ olive oil: 50:50) weekly. From the fourth week of starting injection, 6 nude mice were sacrificed every two weeks by spine dislocation to identify the degree of fibrosis by hematoxylin and eosin (H\&E) and Sirius staining. Further, $\alpha$-SMA was detected using immunohistochemistry till the tenth week.

\section{Establishment of the nude mouse with liver fibrosis bearing orthotopic xenograft}

Male BALB/c nu/nu mice (4-6 weeks old; $n=20$ ) bearing orthotopic xenografts from HCCLM3 were randomly divided into four groups. Three days later, two groups were subcutaneously injected with $\mathrm{CCl}_{4}$ ( $5 \mu \mathrm{l} / \mathrm{g} /$ week), the other two were treated with equal dose of olive oil. At the same time, one group chosen from the $\mathrm{CCl}_{4}$ treated group and the other group from olive oil groups were administered SYY through the drinking medium ( $2 \mathrm{~g} / \mathrm{kg} /$ day). After 6 weeks, all nude mice were sacrificed and tumor volume and the degree of fibrosis were evaluated.

\section{Establishment of the nude mouse bearing subcutaneous tumor with fibrosis background}

Male BALB/c nu/nu mice (4-6 weeks old; $n=20$ ) were divided into four groups. Each group of nude mice were subcutaneously injected with different number $\left(1 \times 10^{6}, 5 \times 10^{5}, 1 \times 10^{5}, 5 \times 10^{4}\right)$ of highly metastatic HCCLM3 cells $(0.1 \mathrm{ml})$. After 12 weeks, following the mice sacrifice the relationship between tumor growth and the number of tumor cells was evaluated.
Male BALB/c nu/nu mice (4-6 weeks old; $n=25$ ) were divided into five groups. Four groups of nude mice were subcutaneously injected with mixed cells (different number of HCCLM3 cells $\left(0.1 \mathrm{ml}, 1 \times 10^{6}, 5 \times 10^{5}\right.$, $1 \times 10^{5}, 5 \times 10^{4}$, ) and human hepatic stellate cells $(0.1 \mathrm{ml}$ LX2, $\left.1 \times 10^{5}\right)$. The other group was just injected with LX2 cells $\left(2 \times 10^{5}, 0.2 \mathrm{ml}\right)$. After 12 weeks, all the mice were sacrificed by cervical dislocation and the subcutaneous tumor formation was evaluated.

Male BALB/c nu/nu mice (4-6 weeks old; $n=6)$ were divided into two groups. These mice were subcutaneously injected with mixed cells (HCCLM3GFP cells $\left(5 \times 10^{5}\right)$ and LX2 cells $\left(5 \times 10^{5}\right)$. One group was treated with SYY for 6 weeks, the other group without intervention. After 6 weeks, the tumor volume was evaluated. Lung metastases were also evaluated by fluorescence microscopy. Then taking two groups of mice, treated in the same way, survival status was determined.

\section{Statistical analysis}

Statistical analysis was carried out using SPSS software version 10.0 for Windows software (SPSS, Chicago, IL, USA). As all groups showed normal distribution, group differences were analyzed using parametric statistical methods, paired independent sample $t$-tests following one-way ANOVA. Data were presented as mean \pm standard deviation, $P<0.05$ was considered statistically significant.

\section{ACKNOWLEDGMENTS}

This research project was supported in part by Key project of Ningxia Natural Science Foundation (NZ15130); The Foundation of China National '211' Project for Higher Education (No. 2007-353); The Postdoctoral Science Foundation of China (2015M570330).

\section{CONFLICTS OF INTEREST}

The authors declare that they have no competing interests.

\section{Authors' contributions}

YB, QAJ, ZGR, TCX, Quan-BaoZ, KZZ, Qiang-BoZ, YY, HT, LXQ and ZYT contributed to the study design, analysis, and interpretation of data. ZYT and ZGR conceived the study. YB and QAJ performed the experiments and drafted the manuscript. TCX and Quan-BaoZ participated in the construction of oxaliplatin-resistant hepatocellular carcinoma cell lines. KZZ and Qiang-BoZ participated in the establishment of the nude mouse model. YY, HT and LXQ participated in statistical analysis. ZYT carried out the revision and provided important suggestions. All authors approved the final manuscript. 


\section{REFERENCES}

1. Jemal A, Bray F, Center MM, Ferlay J, Ward E, Forman D. Global cancer statistics. CA Cancer J Clin. 2011; 61:69-90.

2. Farazi PA, DePinho RA. Hepatocellular carcinoma pathogenesis: from genes to environment. Nat Rev Cancer. 2006; 6:674-687.

3. Neaud V, Faouzi S, Guirouilh J, Le Bail B, Balabaud C, Bioulac-Sage P, Rosenbaum J. Human hepatic myofibroblasts increase invasiveness of hepatocellular carcinoma cells: evidence for a role of hepatocyte growth factor. Hepatology. 1997; 26:1458-1466.

4. Wynn TA. Cellular and molecular mechanisms of fibrosis. J Pathol. 2008; 214:199-210.

5. Sokolovic A, Sokolovic M, Boers W, Elferink RP, Bosma PJ. Insulin-like growth factor binding protein 5 enhances survival of LX2 human hepatic stellate cells. Fibrogenesis Tissue Repair. 2010; 3:3.

6. Kalluri R, Zeisberg M. Fibroblasts in cancer. Nat Rev Cancer. 2006; 6:392-401.

7. Ostman A, Augsten M. Cancer-associated fibroblasts and tumor growth - bystanders turning into key players. Curr Opin Genet Dev. 2009; 19:67-73.

8. Wang W, Li Q, Yamada T, Matsumoto K, Matsumoto I, Oda M, Watanabe G, Kayano Y, Nishioka Y, Sone S, et al. Crosstalk to stromal fibroblasts induces resistance of lung cancer to epidermal growth factor receptor tyrosine kinase inhibitors. Clin Cancer Res. 2009; 15:6630-6638.

9. Rasanen K, Vaheri A. Activation of fibroblasts in cancer stroma. Exp Cell Res. 2010; 316:2713-2722.

10. Studebaker AW, Storci G, Werbeck JL, Sansone P, Sasser AK, Tavolari S, Huang T, Chan MW, Marini FC, Rosol TJ, et al. Fibroblasts isolated from common sites of breast cancer metastasis enhance cancer cell growth rates and invasiveness in an interleukin-6-dependent manner. Cancer Res. 2008; 68:9087-9095.

11. Jia QA, Wang ZM, Ren ZG, Bu Y, Xie XY, Wang YH, Zhang L, Zhang QB, Xue TC, Deng LF, et al. Herbal compound "Songyou Yin" attenuates hepatoma cell invasiveness and metastasis through downregulation of cytokines secreted by activated hepatic stellate cells. BMC Complement Altern Med. 2013; 13:89.

12. Amann T, Bataille F, Spruss T, Muhlbauer M, Gabele E, Scholmerich J, Kiefer P, Bosserhoff AK, Hellerbrand C. Activated hepatic stellate cells promote tumorigenicity of hepatocellular carcinoma. Cancer Sci. 2009; 100:646-653.

13. Mazzocca A, Fransvea E, Dituri F, Lupo L, Antonaci S, Giannelli G. Down-regulation of connective tissue growth factor by inhibition of transforming growth factor beta blocks the tumor-stroma cross-talk and tumor progression in hepatocellular carcinoma. Hepatology. 2010; 51:523-534.

14. Moreira RK. Hepatic stellate cells and liver fibrosis. Arch Pathol Lab Med. 2007; 131:1728-1734.
15. Lee JS, Kim JH. [The role of activated hepatic stellate cells in liver fibrosis, portal hypertension and cancer angiogenesis]. Korean J Hepatol. 2007; 13:309-319.

16. Jiang C, Lee HJ, Li GX, Guo J, Malewicz B, Zhao Y, Lee EO, Lee JH, Kim MS, Kim SH, et al. Potent antiandrogen and androgen receptor activities of an Angelica gigas-containing herbal formulation: identification of decursin as a novel and active compound with implications for prevention and treatment of prostate cancer. Cancer Res. 2006; 66:453-463.

17. Bonham M, Arnold H, Montgomery B, Nelson PS. Molecular effects of the herbal compound PC-SPES: identification of activity pathways in prostate carcinoma. Cancer Res. 2002; 62:3920-3924.

18. Cheng YL, Chang WL, Lee SC, Liu YG, Chen CJ, Lin SZ, Tsai NM, Yu DS, Yen CY, Harn HJ. Acetone extract of Angelica sinensis inhibits proliferation of human cancer cells via inducing cell cycle arrest and apoptosis. Life Sci. 2004; 75:1579-1594.

19. Tsai NM, Lin SZ, Lee CC, Chen SP, Su HC, Chang WL, Harn HJ. The antitumor effects of Angelica sinensis on malignant brain tumors in vitro and in vivo. Clin Cancer Res. 2005; 11:3475-3484.

20. Wang X, Wei Y, Yuan S, Liu G, Lu Y, Zhang J, Wang W. Potential anticancer activity of tanshinone IIA against human breast cancer. Int J Cancer. 2005; 116:799-807.

21. Hong JH, Ahn KS, Bae E, Jeon SS, Choi HY. The effects of curcumin on the invasiveness of prostate cancer in vitro and in vivo. Prostate Cancer Prostatic Dis. 2006; 9:147-152.

22. Kunnumakkara AB, Guha S, Krishnan S, Diagaradjane P, Gelovani J, Aggarwal BB. Curcumin potentiates antitumor activity of gemcitabine in an orthotopic model of pancreatic cancer through suppression of proliferation, angiogenesis, and inhibition of nuclear factor-kappaB-regulated gene products. Cancer Res. 2007; 67:3853-3861.

23. Tamvakopoulos C, Dimas K, Sofianos ZD, Hatziantoniou S, Han Z, Liu ZL, Wyche JH, Pantazis P. Metabolism and anticancer activity of the curcumin analogue, dimethoxycurcumin. Clin Cancer Res. 2007; 13:1269-1277.

24. Chang JY, Chang CY, Kuo CC, Chen LT, Wein YS, Kuo YH. Salvinal, a novel microtubule inhibitor isolated from Salvia miltiorrhizae Bunge (Danshen), with antimitotic activity in multidrug-sensitive and -resistant human tumor cells. Mol Pharmacol. 2004; 65:77-84.

25. Yim D, Singh RP, Agarwal C, Lee S, Chi H, Agarwal R. A novel anticancer agent, decursin, induces G1 arrest and apoptosis in human prostate carcinoma cells. Cancer Res. 2005; 65:1035-1044.

26. Lee HJ, Lee EO, Rhee YH, Ahn KS, Li GX, Jiang C, Lu J, Kim SH. An oriental herbal cocktail, ka-mi-kae-kyuk-tang, exerts anti-cancer activities by targeting angiogenesis, apoptosis and metastasis. Carcinogenesis. 2006; 27:2455-2463. 
27. Singh RP, Agarwal R. Mechanisms of action of novel agents for prostate cancer chemoprevention. Endocr Relat Cancer. 2006; 13:751-778.

28. Lee SM, Li ML, Tse YC, Leung SC, Lee MM, Tsui SK, Fung KP, Lee CY, Waye MM. Paeoniae Radix, a Chinese herbal extract, inhibit hepatoma cells growth by inducing apoptosis in a p53 independent pathway. Life Sci. 2002; 71:2267-2277.

29. Yano H, Mizoguchi A, Fukuda K, Haramaki M, Ogasawara S, Momosaki S, Kojiro M. The herbal medicine sho-saiko-to inhibits proliferation of cancer cell lines by inducing apoptosis and arrest at the G0/G1 phase. Cancer Res. 1994; 54:448-454.

30. Huang XY, Wang L, Huang ZL, Zheng Q, Li QS, Tang ZY. Herbal extract "Songyou Yin" inhibits tumor growth and prolongs survival in nude mice bearing human hepatocellular carcinoma xenograft with high metastatic potential. J Cancer Res Clin Oncol. 2009; 135:1245-1255.

31. Xiong W, Ren ZG, Qiu SJ, Sun HC, Wang L, Liu BB, Li QS, Zhang W, Zhu XD, Liu L, et al. Residual hepatocellular carcinoma after oxaliplatin treatment has increased metastatic potential in a nude mouse model and is attenuated by Songyou Yin. BMC Cancer. 2010; 10:219.

32. Jia QA, Ren ZG, Bu Y, Wang ZM, Zhang QB, Liang L, Jiang XM, Tang ZY. Herbal Compound "Songyou Yin" Renders Hepatocellular Carcinoma Sensitive to Oxaliplatin through Inhibition of Stemness. Evid Based Complement Alternat Med. 2012; 2012:908601.

33. Fattovich G, Stroffolini T, Zagni I, Donato F. Hepatocellular carcinoma in cirrhosis: incidence and risk factors. Gastroenterology 2004. 127:S35-50.

34. Levesque JP. A niche in a dish: pericytes support HSC. Blood. 2013; 121:2816-2818.
35. Ahsan MK, Mehal WZ. Activation of adenosine receptor A2A increases HSC proliferation and inhibits death and senescence by down-regulation of p53 and Rb. Front Pharmacol. 2014; 5:69.

36. Koukourakis MI, Giatromanolaki A, Harris AL, Sivridis E. Comparison of metabolic pathways between cancer cells and stromal cells in colorectal carcinomas: a metabolic survival role for tumor-associated stroma. Cancer Res. 2006; 66:632-637.

37. Iredale JP, Bataller R. Identifying molecular factors that contribute to resolution of liver fibrosis. Gastroenterology. 2014; 146:1160-1164.

38. Visan I. Signals for HSC emergence. Nat Immunol. 2014; $16: 12$.

39. Pietras K, Ostman A. Hallmarks of cancer: interactions with the tumor stroma. Exp Cell Res. 2010; 316:1324-1331.

40. Bhowmick NA, Neilson EG, Moses HL. Stromal fibroblasts in cancer initiation and progression. Nature. 2004; 432:332-337.

41. Fu L, Zhang C, Zhang LY, Dong SS, Lu LH, Chen J, Dai Y, Li Y, Kong KL, Kwong DL, et al. Wnt2 secreted by tumour fibroblasts promotes tumour progression in oesophageal cancer by activation of the Wnt/beta-catenin signalling pathway. Gut. 2011; 60:1635-1643.

42. Yuan SL, Huang RM, Wang XJ, Song Y, Huang GQ. Reversing effect of Tanshinone on malignant phenotypes of human hepatocarcinoma cell line. World J Gastroenterol. 1998; 4:317-319.

43. Tin MM, Cho CH, Chan K, James AE, Ko JK. Astragalus saponins induce growth inhibition and apoptosis in human colon cancer cells and tumor xenograft. Carcinogenesis. 2007; 28:1347-1355. 